\title{
Quantum Walks on Hypergraphs
}

\section{Przemysław Sadowski ${ }^{1}$ • Łukasz Pawela ${ }^{1}$ (D) . Paulina Lewandowska ${ }^{1,2}$. Ryszard Kukulski ${ }^{1,2}$}

Received: 27 January 2019 / Accepted: 8 July 2019 / Published online: 21 August 2019

(C) The Author(s) 2019

\begin{abstract}
In this work we introduce the concept of a quantum walk on a hypergraph. We show that the staggered quantum walk model is a special case of a quantum walk on a hypergraph.
\end{abstract}

Keywords Quantum walks · Hypergraphs

\section{Introduction}

Quantum walks may be seen as an extension of the classical random walks into the quantum realm. There is, however, one key difference. In the classical setting the randomness is built-into the process. In the quantum case, the entire process is unitary, hence deterministic and even reversible. The randomness comes only from the random nature of quantum measurements.

During the last two decades the field of quantum walks has received a lot of attention from the scientific community. One of the earliest studies are the works by Aharonov [1] and Kempe [2]. Soon afterwards the possibility for algorithmic applications was shown [3]. One notable application is the fact that Grover's search algorithm [4] can be represented as a quantum walk. Another approach to database lookup is the quantum spatial search algorithm [5]. Finally, nontrivial results in the field of quantum games can be obtained even with a simple walk on a cycle [6], and some more exotic problems like the Parrondo paradox can be modeled as a quantum walk [7].

Since these seminal works a lot of different approaches to the concept of a quantum walk have emerged. We should note here the open quantum walk model $[8,9]$. This model can be summarized as follows. Imagine we have a particle moving on a graph. The particle has a quantum state associated with it. With each transition from one vertex to another, the state is modified according to some quantum operation. The only restriction here is that all the operations associated with some vertex must sum to a proper quantum channel. There was a lot effort put into studying this approach. We should mention here various asymptotic

Łukasz Pawela

lpawela@iitis.pl

1 Institute of Theoretical and Applied Informatics, Polish Academy of Sciences, ulica Bałtycka 5, 44-100, Gliwice, Poland

2 Institute of Mathematics, University of Silesia, ul. Bankowa 14, 40-007, Katowice, Poland 
Table 1 Summary of existing quantum walk models. The aim of this work is to find the model which fills the gap denoted by the question mark

\begin{tabular}{lll}
\hline Quantum walks summary & \\
\hline Unitary & Reflection & Arbitrary \\
\hline Edges & Szegedy & coined \\
Cliques & staggered & $?$ \\
\hline
\end{tabular}

By "range" we mean where the unitaries involved in the model act

results for this model [10, 11], hitting times studies [12] and potential applications of this model in quantum modeling of biological structures [13]

Another model which deserves mention is the quantum stochastic walk [14]. This approach is based on the Gorini-Kossakowski-Sudarshan-Lindblad [15, 16] master equation. It allows to smoothly interpolate between classical and quantum walks as well as gives raise to some new dynamics. The asymptotic behavior of this model has been extensively studied $[17,18]$.

Finally, there has been a lot of effort put into the extension of the standard unitary quantum walk. Let us note here the Szegedy walk model [19] which allows for quantization of arbitrary Markov chain based algorithms. One of the most prominent example of usage of this model is the quantum Page Rank algorithm [20]. Another example of such modification is the staggered walk model introduced by Portugal et al. [21-23]. This model has applications in quantum search algorithms [24].

In this work we introduce a novel concept - quantum walks on hypergraphs. Our main motivation is presented in Table 1 . In there, we show how the currently developed quantum walk models are constructed. The goal of this work is to fill the part represented by the question mark. For this purpose we introduce a model of a quantum walk on a hypergraph. For the sake of brevity we will call this the hyperwalk model. Next, we introduce methods of comparing models of quantum walks. These are shown in Definition 5 and Definition 6. This allows us to arrive at the main result of this work, shown in Theorem 1 which states that the hyperwalk model indeed introduces new dynamics.

This work is organized as follows. In Section 2 we recall well-established quantum walk models. Section 3 introduces our model-quantum walk on a hypergraph, which for the sake of brevity we will call a hyperwalk. Next, in Section 4 how our model relates to other quantum walks. Finally, in Section 5 final conclusions are drawn.

\section{Walks}

\subsection{The Coined Quantum Walk}

Here we introduce the coined quantum walk model. We start with a simple walk on a cycle. Later we we will discuss the scattering walk model which will allow to extend the concepts presented here to arbitrary graphs.

In the simplest case of a discrete time coined quantum walk on a cycle the model is defined using a bipartite system $\mathcal{H}_{C} \otimes \mathcal{H}_{P}$. Here $\mathcal{H}_{C}$ is a two-dimensional Hilbert space with basis $\{|0\rangle,|1\rangle\}$, called the coin space, and $\mathcal{H}_{P}=\operatorname{span}(\{|n\rangle: n \in\{0, \ldots, N-1\}\})$ is the position space. Let us introduce two unitary operations: $C$, a coin operator acting on the 
Table 2 The probability of occupying site $i$ after $T$ steps of the quantum walk on the cycle with $N=7$ vertices for the initial state $\left|\psi_{0}\right\rangle=|0\rangle \otimes|0\rangle$

\begin{tabular}{|c|c|c|c|c|c|c|c|}
\hline$T$ & 4 & 5 & 6 & 0 & 1 & 2 & 3 \\
\hline 0 & & & & 1 & & & \\
\hline 1 & & & $\frac{1}{2}$ & & $\frac{1}{2}$ & & \\
\hline 2 & & $\frac{1}{4}$ & & $\frac{1}{2}$ & 2 & $\frac{1}{4}$ & \\
\hline 3 & $\frac{1}{8}$ & & $\frac{1}{8}$ & & $\frac{5}{8}$ & & $\frac{1}{8}$ \\
\hline 4 & $\frac{1}{16}$ & $\frac{1}{8}$ & & $\frac{1}{8}$ & & $\frac{5}{8}$ & $\frac{8}{16}$ \\
\hline 5 & $\frac{5}{32}$ & $\frac{1}{32}$ & $\frac{1}{8}$ & & $\frac{1}{8}$ & $\frac{1}{32}$ & $\frac{17}{32}$ \\
\hline
\end{tabular}

space $\mathcal{H}_{C}$ and $S$, a shift operator acting on the space $\mathcal{H}_{C} \otimes \mathcal{H}_{P}$. Every step of the evolution, $U$, is a composition of the coin and shift operators

$$
\left|\psi_{t+1}\right\rangle=S\left(C \otimes \mathbb{1}_{N}\right)\left|\psi_{t}\right\rangle,
$$

where $\left|\psi_{0}\right\rangle \in \mathcal{H}_{\mathcal{C}} \otimes \mathcal{H}_{\mathcal{P}}$ is some initial state. In this setup, the shift operator is defined by

$$
S=|0\rangle\left\langle 0\left|\otimes \sum_{i=0}^{N-1}\right|(i+1)_{N}\right\rangle\langle i|+| 1\rangle\left\langle 1\left|\otimes \sum_{i=0}^{N-1}\right|(i-1)_{N}\right\rangle\langle i|,
$$

where $N$ is the length of the cycle. The unitary transformation $C$ is arbitrary. By modifying the coin operator we can get different dynamics and probability distribution in the quantum walk.

Example 1 Consider the coin operator

$$
C=\frac{1}{\sqrt{2}}\left(\begin{array}{ll}
1 & \mathrm{i} \\
\mathrm{i} & 1
\end{array}\right) \text {. }
$$

This operator is balanced, because after first step we have

$$
S\left(C \otimes \mathbb{1}_{N}\right)(|0\rangle \otimes|0\rangle)=\frac{1}{\sqrt{2}}|0\rangle \otimes|1\rangle+\frac{\mathrm{i}}{\sqrt{2}}|1\rangle \otimes|N-1\rangle .
$$

After measuring this state in the standard basis of $\mathcal{H}_{C} \otimes \mathcal{H}_{P}$ we get one of two states $\{|0\rangle \otimes|1\rangle,|1\rangle \otimes|N-1\rangle\}$ each with probability equal to $\frac{1}{2}$.

The probability distributions for this particular model after the first few steps are shown in Table 2. As the initial state we choose $\left|\psi_{0}\right\rangle=|00\rangle$, the cycle length is $N=7$.

\subsection{Scattering Quantum Walk}

Let's consider a graph $G=(V, E)$ [25], where $V$ is a set of vertices and $E \subseteq V \times V$ is a set of edges. We say an element $i \in V$ is connected with element $j \in V$ when $(i, j) \in E$. We will denote this by $i \sim j$. In order to model a coined quantum walk on a graph which is not regular, we must modify this simple approach considerably. This can be achieved via the scattering walk approach. In this case, we introduce separate coin operators for every vertex $v$ of the graph $G$. Let us denote the degree fo each vertex $i$ as $d_{i}$. We introduce a coin operator $C_{i}$ for each vertex. Hence the entire coin operator is vertex as $d_{i}$, we have that each coin operator $C_{i}$ acts on $\mathbb{C}^{d_{i}}$. The entire space is

$$
\mathcal{X}=\mathbb{C}^{d_{1}} \oplus \ldots \oplus \mathbb{C}^{d_{N}}
$$


The shift operator performs the scattering on a vertex $i$ given by formula

$$
S|i, j\rangle=|j, i\rangle .
$$

Let us consider a particle coming to vertex $j$ from some vertex $i$, i.e. moving along the edge $(i, j)$. It becomes scattered after the shift operation, meaning that with equal probability it gets transfered to all other edges outgoing from $j$ and gets reflected back to $i$ along the $(j, i)$ edge, provided it exists in $G$. Such a walk is defined by unitary operation $U$, that for every vertex $i$ fullfils [26]

$$
U|i, j\rangle=r_{i}|j, i\rangle+t_{i} \sum_{v \sim j, v \neq i}|j, v\rangle .
$$

Of course unitarity requires $\left|r_{i}\right|^{2}+\left(\operatorname{deg}\left(v_{i}\right)-1\right)\left|t_{i}\right|^{2}=1$ and the entire evolution is given by this formula. In the case of a directed graph $G$, we need to remember that each edge $(i, j)$ can be seen as two directed edges.

For regular structures, i.e. all the degrees of all vertices are equal, this simplifies to

$$
C=C_{0}^{\oplus N} \cong C_{0} \otimes \mathbb{1}_{N}
$$

where $C_{0}$ is the coin operator for vertex $v_{0}$, Let consider as an example the simple case when $r_{i}=1$ for every $i$. In this case the shift operator for each edge subspace $\mathcal{X}_{i, j}=$ $\operatorname{span}(\{|i, j\rangle,|j, i\rangle\})$ acts as $\sigma_{x}$ - Pauli-X operator. Using a different basis, we may write the space $\mathcal{X}$ as $\mathcal{X}_{E}=\oplus_{i \sim j} \mathcal{X}_{i, j}$. Then, the shift operator takes the form

$$
S=\sigma_{x} \otimes \mathbb{1}_{|E|} \cong \sigma_{x}^{\oplus|E|} .
$$

The shift operator is a block operator in

$$
\mathcal{X}=\bigoplus_{i \sim j} \operatorname{span}(\{|i, j\rangle,|j, i\rangle\})
$$

and the coin operator is a block operator in

$$
\mathcal{Y}=\bigoplus_{j \in V} \operatorname{span}\left(\{|i, j\rangle\}_{i \sim j}\right)
$$

Example 2 Let us introduce the following example. Consider the scattering quantum walk on a complete bipartite graph $K_{3,2}$, where $V=V_{1} \cup V_{2}=\{1,2,3\} \cup\{a, b\}$. We define the coin operator as

$$
C=F_{2} \oplus F_{2} \oplus F_{2} \oplus F_{3} \oplus F_{3},
$$

Table 3 The probability of being found at position $i$ after $T$ steps of the quantum walk on the complete bipartite graph $K_{3,2}$ for the initial state $\left|\psi_{0}\right\rangle=|1, a\rangle$

\begin{tabular}{lccccc}
\hline$i$ & 1 & 2 & 3 & $\mathrm{a}$ & $\mathrm{b}$ \\
\hline 0 & 1 & & & $\frac{1}{2}$ & $\frac{1}{2}$ \\
1 & $\frac{1}{3}$ & $\frac{1}{3}$ & $\frac{1}{3}$ & & \\
2 & 1 & & & 1 & $\frac{1}{2}$ \\
3 & & & & $\frac{1}{2}$ \\
4 & & &
\end{tabular}


where $F_{n}$ is $n$-dimensional Fourier matrix. The shift operator is defined by (6). To illustrate behavior of scattering quantum walk for first five steps with the initial state $\left|\psi_{0}\right\rangle=|1, a\rangle$, we present the probability distribution of each step in Table 3.

Additionally we allow cases in which the operator $C$ changes in time in a cyclic manner and call such model a generalized coined walk model.

\subsection{The Szegedy Walk Model}

The Szegedy walk model was first introduced in [19] as a model which allows quantization of arbitrary Markov chain based algorithms. The model is as follows. We start with undirected graph $G_{C}=G(V, E)$ and we set a bipartite graph $G_{S}=G\left(V \cup V^{\prime}, F\right)$ where $V^{\prime}$ is the same as $V$ with all elements primed. As for the edges we have $\left(i, j^{\prime}\right) \in F$ if and only if $(i, j) \in E$. The evolution is given by the unitary operators $U_{1}, U_{2} U_{1}, U_{1} U_{2} U_{1}, \ldots$ acting on the space $\operatorname{span}\left(\left\{\left|x, y^{\prime}\right\rangle: x \in V, y^{\prime} \in V^{\prime}\right\}\right)$.

We define reflections

$$
\begin{aligned}
& U_{1}=2 \sum_{V}\left|d_{v}\right\rangle\left\langle d_{v}\right|-\mathbb{1}, \\
& U_{2}=2 \sum_{V}\left|\bar{d}_{v}\right\rangle\left\langle\bar{d}_{v}\right|-\mathbb{1},
\end{aligned}
$$

and unit vectors

$$
\begin{aligned}
& \left|d_{v}\right\rangle=|v\rangle \otimes \sum_{w \sim v} a_{v, w}|w\rangle, \\
& \left|\bar{d}_{v}\right\rangle=\sum_{w \sim v} a_{v, w}|w\rangle \otimes|v\rangle,
\end{aligned}
$$

where $a_{v, w}$ are complex constants.

Usually, the unitary operators driving the evolution for the Szegedy walk model are chosen as presented above. In our work, we assume that unitary operators can be chosen arbitrary with only assumption of respecting the graph structure i.e. the movement between not connected vertices is forbidden.

\subsection{The Staggered Walk}

To formally introduce the staggered walk model, we first introduce the following definitions. We will follow the definitions introduced by Portugal et al. [21]

Definition 1 A partition of a set $\mathrm{A}$ is a collection $\alpha=\left\{p_{k}\right\}_{k}$ of subsets of A, $p_{k} \subset A$, such that $\bigcup_{k} p_{k}=A$ and $p_{k} \cap p_{k^{\prime}}=\emptyset$ for $k \neq k^{\prime}$.

Definition 2 A partition of a graph $G=(V, E)$ is a partition of V such that each $p_{k}$ forms a clique or is a single vertex. We will call $p_{k}$ a polygon.

Note that this definition allows for a polygon to contain a single vertex. The staggered quantum walk on a graph is defined using at least one graph partition. 
Definition 3 Given a graph $G(V, E)$ and its n partitions $\alpha_{1}, \ldots, \alpha_{n}, \alpha_{k}=\left\{p_{k, i}\right\}_{i}$, for $k=$ $1, \ldots, n$, the staggered quantum walk is defined by the evolution operator $U \in \mathrm{U}\left(\mathcal{X}^{V}\right)$, where $\mathcal{X}^{V}=\mathbb{C}^{|V|}$ :

where

$$
U=U_{n} \ldots U_{2} U_{1}
$$

$$
U_{k}=2 \sum_{i=1}^{\left|\alpha_{k}\right|}\left|d_{k, i}\right\rangle\left\langle d_{k, i}\right|-\mathbb{1}_{\mathcal{X}^{V}} .
$$

The states $\left|d_{k, i}\right\rangle$ are:

$$
\left|d_{k, i}\right\rangle=\sum_{j \in p_{k, i}} a_{k, j}|j\rangle
$$

where $a_{k, j}$ are complex amplitudes.

For the staggered quantum walk model we assume, that unitary operators can be also chosen arbitrarily.

\section{Hyperwalk Model}

In this section we introduce the concept of quantum walks on hypergraph networks along with some intuitions and call it the quantum hyperwalk model. We denote hypergraph by $H=(V, E)$ [27], where $V$ is a set of vertices as in the traditional graph case and $E$ is the set of hyperedges defined as a collection of subsets of vertices $E \subseteq 2^{V}$.

Now we want to emphasize that the coined quantum walk model can be described as a composition of two operators that take block operator form with respect to two different partitions of the computational basis. The main restriction in the model is that the partitions corresponding to the edges of the graph always consists of sets with two basis states. We aim at loosening this restriction and developing a quantum walk model suitable for hypergraphs in the sense that a partition of a hypergraph $H=(V, E)$ is a tesselation of the basis states $\{|v, e\rangle: v \in V, e \in E\}$.

Definition 4 We define a hyperwalk on a hypergraph $H(V, E)$ as a composition $U^{E} U^{V}$ of two unitary operators: $U^{V}$ and $U^{E}$ on the space $\mathcal{X}=\operatorname{span}(\{|v, e\rangle: e \in E \wedge v \in e\})$, where

$$
\begin{aligned}
& U^{V}=\sum_{v \in V} C_{v}, \\
& U^{E}=\sum_{e \in E} S_{e},
\end{aligned}
$$

for $C_{v}$ being a coin operator for a fixed vertex $v$ acting on $\operatorname{span}\left(\{|v, e\rangle\}_{e \in E}\right)$ and $S_{e}$ being a shift operator for a fixed edge $e$ acting on $\operatorname{span}\left(\{|v, e\rangle\}_{v \in e}\right)$.

We also introduce a generalized version of this model. By a generalized hyperwalk we mean an instance of a hyperwalk for which the underlying unitaries change over time.

Here we present an example of construction of such walk.

Example 3 We define $C_{v}=\mathbb{1}_{\operatorname{deg}(v)}-2\left|\psi_{v}\right\rangle\left\langle\psi_{v}\right|$ for $\left|\psi_{v}\right\rangle=\frac{1}{\sqrt{\operatorname{deg}(v)}} \sum_{\{e: v \in e\}}|v, e\rangle$ and $S_{e}=\mathbb{1}_{|e|}-2\left|\psi_{e}\right\rangle\left\langle\psi_{e}\right|$ for $\left|\psi_{e}\right\rangle=\frac{1}{\sqrt{|e|}} \sum_{v \in e}|v, e\rangle$. This is a hypergraph generalization of the Grover's walk. Let us note that for a 2-regular hypergraph, i.e. an ordinary graph, we 
Fig. 1 Example of hyperwalk.

We have the following 3

hyperedges: $a=\{A, B, C\}$,

$b=\{A, B\}, c=\{C, D\}$

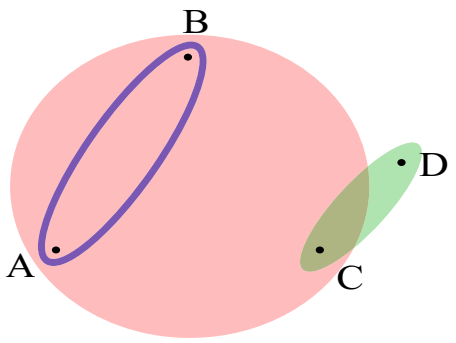

obtain $S_{e}$ which are two dimensional Grover's diffusion operator that are equal to $\sigma_{x}$. This shows that our model recovers the proper behaviour for a hypergraph which reduces to an ordinary graph.

Example 4 The idea of a hyperwalk gives the possibility to implement walks on directed graphs. The basic way to ensure that computation performed with use of directed connections is reversible (unitary) is to ensure that for each vertex the number of directed inputs and outputs is the same. In order to satisfy this condition for a finite graph the directed connections must contain loops, which may be seen as hyperedges. Thus, we define a quantum walk with directed edges as

$$
S_{E}=\sum_{\left(v_{1}, \ldots, v_{l_{e}}\right)=e \in E} \sum_{i=1}^{l_{e}}\left|v_{i+1}, e\right\rangle\left\langle v_{i}, e\right|,
$$

where $E$ is a set of edges defined as ordered sequences of vertices. In the case of 2-element edges we recover the canonical shift operator. For a hyperedge we obtain cyclic shift among the loop constructed by this edge.

Example 5 Assume we have hypergraph introduced as in Fig. 1. We consider the hyperwalk on this hypergraph. We choose the coin operator as

$$
C=F_{2} \oplus F_{2} \oplus F_{2} \oplus \mathbb{1}_{1},
$$

where $F_{n}$ is the $n$-dimensional Fourier matrix. The shift operator is

$$
S=U\left(F_{2} \oplus F_{3} \oplus F_{2}\right) U^{\ddagger},
$$

where $U$ is a permutation matrix which permutes second the and third columns. We illustrate behavior of hyperwalk for the first five steps with the initial state $\left|\psi_{0}\right\rangle=|A, a\rangle$. The resulting probability distribution after each step are shown in Table 4.

Table 4 The numerical approximation of probability of founding state in position $i$ after $T$ steps of the quantum walk on hypergraph Fig. 1 for the initial state $\left|\psi_{0}\right\rangle=|A, a\rangle$

\begin{tabular}{lllll}
\hline & A & B & C & D \\
\hline 0 & 1 & & & \\
1 & 0.42 & 0.42 & 0.16 & 0.04 \\
2 & 0.88 & 0.02 & 0.06 & 0.08 \\
3 & 0.43 & 0.43 & 0.06 & 0.1 \\
4 & 0.86 & 0.01 & 0.03 & 0.03 \\
5 & 0.38 & 0.42 & 0.17 & \\
\hline
\end{tabular}


The hyperwalk model can be seen as a natural generalization of the coined walk model. This generality comes from two facts. First of all, by using hyperedges, it is possible to construct higher dimensional space of basis states than in coined walk. Second, loosening the restriction of shift operator to be a permutation matrix, gives us additional dynamics in the constructed space.

\section{Relations Between Models}

It is crucial that the hyperwalk allows us to model quantum walks discussed in previous sections. Additionally, we would like to keep the number of basis states as small as possible. This is the purpose of this section: to compare walk models discussed in the previous sections. In order to achieve this we introduce two alternative definitions of comparing walks and next we present our results of comparing quantum walk models. Our main results are shown in Theorem 1.

To clarify notation let $Q W_{A}(G,|\psi\rangle, n)$ denote the state after $n$ iterations of discrete-time quantum walk model $A$ on graph $G$ with initial an state $|\psi\rangle$. We also use the notation $\mathcal{P}$ for measurement on vertices, where the probability of finding state $|\psi\rangle$ in vertex $v$ is denoted by $\mathcal{P}(|\psi\rangle)(v)$.

Definition 5 Given two walk models $\mathrm{A}$ and $\mathrm{B}$, we say that model $\mathrm{A}$ is an instance of model B $(A \preceq B)$ when for every $G_{A}\left(V_{1}, E_{1}\right)$, measurement $\mathcal{P}_{A}$ on vertices $V_{1}$ and initial state $\left|\psi_{A}\right\rangle$, there exists $G_{B}\left(V_{2}, E_{2}\right)$, where $V_{1} \subset V_{2}$, measurement $\mathcal{P}_{B}$ on vertices $V_{2}$ and initial state $\left|\psi_{B}\right\rangle$, such that for all $n_{0} \in \mathbb{N}_{0}$, exists $n_{1} \in \mathbb{N}_{0}$, such that we have

$$
\mathcal{P}_{A}\left(Q W_{A}\left(G_{A},\left|\psi_{A}\right\rangle, n_{0}\right)\right)(v)=\mathcal{P}_{B}\left(Q W_{B}\left(G_{B},\left|\psi_{B}\right\rangle, n_{1}\right)\right)(v) .
$$

This definition allows to model a given quantum walk model with another model. Unfortunately, that means it allows us to find equivalence between walk models, which are very loosely based on the underlying structure. Some examples of equivalence of walk models which can be derived from this definition are presented below.

1. Szegedy $\preceq$ coined and coined $\succeq$ Szegedy: In the coined walk model we are given a set of basis states $|i, j\rangle$, where $i, j \in V$ if $(i, j) \in E$ and in Szegedy walk model our basis states are $\left|i, j^{\prime}\right\rangle$ if $(i, j) \in E$. There exists a bijection $\Xi$, that is defined as $\Xi(|i, j\rangle)=\left|i, j^{\prime}\right\rangle$. Now we can assume, that if $C$ is a given operator we take $U_{1}=C$ and $U_{2}$ is constructed as in definition of Szegedy walk model. If $U_{1}$ is given then we put $C=U_{1}$. Then, as we can observe, the equalities are satisfied

$$
\Xi(S C S C|\psi\rangle)=U_{2} \Xi(S C|\psi\rangle)=U_{2} U_{1} \Xi(|\psi\rangle)
$$

The above follows from the fact both space are the same up to labeling. This observation implies that measurements are connected by $P_{S z}=\Xi\left(P_{C}\right)$, hence they are the same.

2. hyperwalk $\preceq$ coined: For a given hypergraph $H(V, E)$ with the evolution operator $U_{H}=U^{E} \bar{U}^{V}$, an initial state $\left|\psi_{0}\right\rangle_{H}=|v, e\rangle$ and a measurement $\mathcal{P}_{H}(|\psi\rangle)(v)=$ $\sum_{e \in E}|\langle v, e \mid \psi\rangle|^{2}$, we create a bipartite graph $G\left(V_{1} \cup V_{2}, F\right)$, where the sets $V_{1}=V$ and $V_{2}=E$ are the partitions of a bipartite graph, and $(v, e) \in F$ if and only if 
$v \in V_{1}$ is contained in an edge $e_{1} \in E$. We construct a space with the basis vectors $\{|v, e\rangle,|e, v\rangle: v \in V, e \in E\}$. We define the shift operator in standard form

$$
\begin{aligned}
& S|v, e\rangle=|e, v\rangle \\
& S|e, v\rangle=|v, e\rangle,
\end{aligned}
$$

and the coin operator as

$$
C=U^{V} \oplus U^{E} \text {. }
$$

Consequently, the evolution in quantum coined walk model with the initial state $\left|\psi_{0}\right\rangle_{C}=|v, e\rangle$ is given by

$$
\begin{aligned}
U_{C} & =S C S C=S C\left(|1\rangle\left\langle 0\left|\otimes U^{V}+\right| 0\right\rangle\langle 1| \otimes V^{E}\right) \\
& =S\left(|1\rangle\left\langle 0\left|\otimes U^{E} U^{V}+\right| 0\right\rangle\langle 1| \otimes U^{V} U^{E}\right)=U^{E} U^{V} \oplus U^{V} U^{E} .
\end{aligned}
$$

Then the measurement should be $\mathcal{P}_{C}(|\psi\rangle)(v)=\sum_{e \in E}|\langle v, e \mid \psi\rangle|^{2}$ and now it is clear that $\mathcal{P}_{H}\left(U_{H}^{n}\left|\psi_{0}\right\rangle_{H}\right)=\mathcal{P}_{C}\left(U_{C}^{n}\left|\psi_{0}\right\rangle_{C}\right)$.

3. generalized hyperwalk $\preceq$ staggered: We are given hypergraph $H(V, E)$ with the evolution operator $U_{G_{H}, k}=\bar{U}_{k}^{E} U_{k}^{V}$, for $k \in\{1, \ldots, K\}$, an initial state $\left|\psi_{0}\right\rangle_{G_{H}}=|v, e\rangle$ and a measurement $\mathcal{P}_{G_{H}}(|\psi\rangle)(v)=\sum_{e \in E}|\langle v, e \mid \psi\rangle|^{2}$. Let us note that we can consider an $N$ dimensional system for staggered walk, where $N$ is the number of basis states in generalized hyperwalk. We can set $W=\{|v, e\rangle: v \in V, e \in E, v \in e\}$ as a set of vertices for graph, which defined staggered walk on it and take initial state $\left|\psi_{0}\right\rangle_{S}=\left|\psi_{0}\right\rangle_{G_{H}}$. We introduce such tessellations for which the unitary matrices $U_{i}^{E}, U_{j}^{V}$ can be treated as evolution operators. The measurement on the staggered walk model works on proper group of vertices i.e. $\mathcal{P}_{S}(|\psi\rangle)(v)=\sum_{|v, e\rangle \in W}|\langle v, e \mid \psi\rangle|^{2}$. It is clear that we obtain the same evolution, because as introduced is this case base states in staggered walk are the same as in generalized hyperwalk model.

4. $\quad$ staggered $\preceq$ generalized coined: We are given graph $G(V, E)$ with $N$ vertices, $V=$ $\{1, \ldots, N\}, k$ tessellations and unitaries $U_{1}, \ldots, U_{k}$. We take an initial state $\left|v_{0}\right\rangle_{S}$ and measurement $\mathcal{P}_{S}(|\psi\rangle)(v)=|\langle v \mid \psi\rangle|^{2}$. We construct a new graph by adding to the set $V$ one vertex $t_{i, j}$ for each polygon $j$ in each tessellation $i$. We connect every newly added vertex associated with some polygon to vertices included in this polygon. Let us denote the basis states by $\left\{\left|v, t_{i}\right\rangle,\left|t_{i}, v\right\rangle\right\}$, where $v \leq N, i \leq k-1$. Here, we omitted the second index in vertices $t_{i, j}$, because parameters $v$ and $i$ are sufficient to determine vertex $t_{i}$ uniquely in state $\left|v, t_{i}\right\rangle$. The generalized coined walk on this graph will be represented by $S C S C_{k-1} \ldots S C S C_{0}$. Here, $S$ is the standard shift operator, $C$ is defined by

$$
\begin{aligned}
& C\left|v, t_{i}\right\rangle=\left|v, t_{(i+1)_{k}}\right\rangle, \\
& C\left|t_{i}, v\right\rangle=\left|t_{i}, v\right\rangle .
\end{aligned}
$$

and $C_{i}$ is defined as

$$
\begin{aligned}
C_{i}\left|v, t_{j}\right\rangle & =\left|v, t_{j}\right\rangle, \\
C_{i}\left|t_{j}, v\right\rangle & =\left|t_{j}, v\right\rangle, \quad j \neq i \\
C_{i}\left|t_{i}, v\right\rangle & =\sum_{w \in V}\left\langle w\left|U_{i+1}\right| v\right\rangle\left|t_{i}, w\right\rangle .
\end{aligned}
$$

The initial state can be chosen to be $\left|\psi_{0}\right\rangle_{G_{C}}=\left|t_{0}, v_{0}\right\rangle$ and the measurement of vertex $v$ is $\mathcal{P}_{G_{C}}(|\psi\rangle)(v)=\sum_{\left|t_{i}, v\right\rangle}\left|\left\langle t_{i}, v \mid \psi\right\rangle\right|^{2}$. To see the equality between both measurements, we start with $\left|v_{0}\right\rangle_{S}$ and after the first step we obtain $U_{1}\left|v_{0}\right\rangle_{S}$ in the staggered walk 
model. Assuming that the first step in the generalized coined walk is given by $S C S C_{0}$, we get

$$
\begin{aligned}
S C S C_{0}\left|t_{0}, v_{0}\right\rangle_{G_{C}} & =S C S \sum_{w \in V}\left\langle w\left|U_{1}\right| v_{0}\right\rangle_{S}\left|t_{0}, w\right\rangle=S C \sum_{w \in V}\left\langle w\left|U_{1}\right| v_{0}\right\rangle_{S}\left|w, t_{0}\right\rangle \\
& =S \sum_{w \in V}\left\langle w\left|U_{1}\right| v_{0}\right\rangle_{S}\left|w, t_{1}\right\rangle=\sum_{w \in V}\left\langle w\left|U_{1}\right| v_{0}\right\rangle_{S}\left|t_{1}, w\right\rangle .
\end{aligned}
$$

Then $\left\langle w\left|U_{1}\right| v_{0}\right\rangle_{S}=\left\langle t_{1}, w\left|S C S C_{0}\right| t_{0}, v_{0}\right\rangle_{G_{C}}$, so finally we get

$$
\mathcal{P}_{G_{C}}\left(S C S C_{k-1} \ldots S C S C_{0}\left|t_{0}, v_{0}\right\rangle_{G_{C}}\right)(v)=\mathcal{P}_{S}\left(U_{k} \ldots U_{1}\left|v_{0}\right\rangle_{S}\right)(v) .
$$

5. generalized coined $\preceq$ coined: For a given graph $G$ with $N$ vertices, $\{1, \ldots, N\}$ with changing in time coins $C_{0}, \ldots, C_{k-1}$, an initial state $\left|\psi_{0}\right\rangle=|v, w\rangle$ and a measurement $\mathcal{P}_{G_{C}}\left(\left|\psi_{0}\right\rangle\right)(v)=\sum_{w \in V}\left|\left\langle v, w \mid \psi_{0}\right\rangle\right|^{2}$. We introduce a new graph with $k N$ vertices $v^{(i)}$, where $v \leq N, i \in 0, \ldots, k-1$. In this graph we have connections only between vertices $v^{(i)}, w^{(i+1)_{k}}$, for $v, w \leq N, i \in 0, \ldots, k-1$ if and only if $v \sim w$ in $G$. This generates new basis states $\left\{\left|v^{(i)}, w^{(i+1)_{k}}\right\rangle,\left|w^{(i+1)_{k}}, v^{(i)}\right\rangle\right\}$. In this model the initial state will be $\left|v^{(0)}, w^{(k-1)}\right\rangle$ and the state will evolve to vertices with higher indexes and eventually come back to vertices with the index zero. This means, we define the coin operator as

$$
\begin{aligned}
C\left|v^{(i)}, w^{(i-1)_{k}}\right\rangle & =\sum_{z \in V}\left\langle v, z\left|C_{i}\right| v, w\right\rangle\left|v^{(i)}, z^{(i+1)_{k}}\right\rangle, \\
C\left|v^{(i)}, w^{(i+1)_{k}}\right\rangle & =\left|v^{(i)}, w^{(i-1)_{k}}\right\rangle .
\end{aligned}
$$

The measurement on the vertex $v$ is given on states associated with $v^{(i)}$ i.e. $\mathcal{P}_{C}\left(\left|\psi_{0}\right\rangle\right)(v)=\sum_{i} \sum_{w \sim v}\left|\left\langle v^{(i)}, w^{(i-1)_{k}} \mid \psi_{0}\right\rangle\right|^{2}$.

After the first iteration in the coined walk model we have

$$
\begin{aligned}
S C\left|\psi_{0}\right\rangle=S C\left|v^{(0)}, w^{(k-1)}\right\rangle & =S \sum_{z \in V}\left\langle v, z\left|C_{0}\right| v, w\right\rangle\left|v^{(0)}, z^{(1)}\right\rangle \\
& =\sum_{z \in V}\left\langle v, z\left|C_{0}\right| v, w\right\rangle\left|z^{(1)}, v^{(0)}\right\rangle .
\end{aligned}
$$

On the other hand, considering the generalized coined walk model gives us

$$
S C_{0}\left|\psi_{0}\right\rangle=S C_{0}|v, w\rangle=\sum_{z \in V}\left\langle v, z\left|C_{0}\right| v, w\right\rangle|z, v\rangle .
$$

We can observe, that the both models give us the same evolution, which implies that the measurement outcomes will be exactly the same.

As it can be seen, according to this definition the models are equivalent. This result should not be surprising, as we are allowed to compare models $A$ and $B$ on graphs with different structures. For the case, when $A \preceq B$, the quantum walk model $B$ does not have to express the idea of random walk on graph $G_{A}$.

For example if we want to change generalized hyperwalk with 2 different distributions on the graph shown in Fig. 1 into the Szegedy walk, we need to take graph with 336 vertices. According to the previous discussion changing the generalized hyperwalk to a staggered walk costs 7 vertices. We see that if we want to put this model to generalized coined walk we should take a graph with 21 nodes and 8 coin operators. In the next step, it is necessary 
to model coined walk on a graph with $8 \times 21=168$ vertices. The last step is cloning of the vertices to obtain Szegedy walk, so we end up with 336 nodes required. Of course there still can exist methods to achieve this result with a smaller number of vertices, but this example is introduced to show problems which can appear. That is why we introduce a new concept of comparing two quantum models.

Definition 6 Let $\mathrm{A}$ and $\mathrm{B}$ be two models of a quantum walk. We say that model $\mathrm{A}$ is strongly an instance of model B $(A \prec B)$ when for all graphs $G_{A}(V, E)$ there exists a graph $G_{B}$, such that the number of basis states in model B is no greater then the number of basis states in model A. Moreover, for all initial states $\left|\psi_{A}\right\rangle$ there exists an initial state $\left|\psi_{B}\right\rangle$, such that for all $n \in \mathbb{N}_{0}$ and $v \in V$, we have

$$
\mathcal{P}_{A}\left(Q W_{A}\left(G_{A},\left|\psi_{A}\right\rangle, n\right)\right)(v)=\mathcal{P}_{B}\left(Q W_{B}\left(G_{B},\left|\psi_{B}\right\rangle, n\right)\right)(v) .
$$

Based on this definition, we show that every staggered walk is an instance of a generalized hyperwalk. Furthermore, we do not need to deeply change the structure of the initial graph in order to obtain this behavior.

Theorem 1 According to Definition 6 every staggered walk is an instance of the generalized hyperwalk.

Proof For a given graph with the staggered walk, defined by unitary matrices $U_{1}, \ldots, U_{n}$, we introduce a hypergraph with the same number of vertices and one hyperedge containing all vertices suitable for generalized hyperwalk. One can see that the spaces for both walks have the same dimensionality hence there exists a bijection between spaces on staggered and generalized coined walk models. So we can assume that the coin operator is constant and it is given by the identity matrix. The shift operator is changing in time in the same manner as the unitary operators $U_{1}, \ldots, U_{n}$ for each tessellations, namely $U_{k}^{E}:=U_{k}$. If the measurement for staggered walk is $\mathcal{P}_{S}(|\psi\rangle)(v)=|\langle v \mid \psi\rangle|^{2}$, then we take $\mathcal{P}_{G H}=|\langle v, e \mid \psi\rangle|^{2}$.

\section{Conclusions}

In this work we introduced a model of quantum walks on hypergraphs and a generalized version of this model. By generalized we mean that the evolution operators associated with the walk might change in time. We introduced two non-equivalent definitions of the case when one quantum walk model is an instance of another model. The first definition of this equivalence allows us to heavily manipulate the underlying graph structure of the walk. Using this definition we shown that hyperwalk model is equivalent to a coin model and the same for the generalized version.

Next, we introduced a stronger version of the equivalence of walk models. In it, we enforce the graph to be a minimal graph necessary for a given model. In this regime we were able to show that a generalized hyperwalk introduces in fact new dynamics. This result completes Table 1 and shows that a quantum walk on a hypergraph is a generalization of the staggered walk model.

Acknowledgments This work was supported by the polish National Science Centre under project numbers 2015/17/B/ST6/01872 (ŁP and PL) and 2016/22/E/ST6/00062 (RK). 
Open Access This article is distributed under the terms of the Creative Commons Attribution 4.0 International License (http://creativecommons.org/licenses/by/4.0/), which permits unrestricted use, distribution, and reproduction in any medium, provided you give appropriate credit to the original author(s) and the source, provide a link to the Creative Commons license, and indicate if changes were made.

\section{References}

1. Aharonov, D., Ambainis, A., Kempe, J., Vazirani, U.: Quantum walks on graphs. In: Proceedings of the thirty-third annual ACM symposium on Theory of computing, pp. 50-59. ACM (2001)

2. Kempe, J.: Quantum random walks: an introductory overview. Contemp. Phys. 44(4), 307-327 (2003)

3. Ambainis, A.: Quantum walks and their algorithmic applications. Int. J. Quantum Inf. 1(04), 507-518 (2003)

4. Grover, L.K.: A fast quantum mechanical algorithm for database search. In: Proceedings of the twentyeighth annual ACM symposium on Theory of computing, pp. 212-219. ACM (1996)

5. Childs, A.M., Goldstone, J.: Spatial search by quantum walk, vol. 70 (2004)

6. Miszczak, J.A., Sadowski, P.: Quantum network exploration with a faulty sense of direction. Quantum Inf. Comput. 14(13\&14), 1238-1250 (2014)

7. Pawela, Ł., Sładkowski, J.: Cooperative quantum Parrondo’s games. Physica D: Nonlinear Phenom. 256, 51-57 (2013)

8. Attal, S., Petruccione, F., Sinayskiy, I.: Open quantum walks on graphs. Phys. Lett. A 376(18), 15451548 (2012)

9. Attal, S., Petruccione, F., Sabot, C., Sinayskiy, I.: Open quantum random walks. J. Stat. Phys. 147(4), 832-852 (2012)

10. Attal, S., Guillotin-Plantard, N., Sabot, C.: Central Limit Theorems for Open Quantum Random Walks and Quantum Measurement Records. In: Annales Henri PoincarÈ, vol. 16, pp. 15-43. Springer (2015)

11. Sadowski, P., Pawela, Ł.: Central Limit theorem for reducible and irreducible open quantum walks. Quantum Inf. Process 15(7), 2725-2743 (2016)

12. Pawela, Ł., Gawron, P., Miszczak, J.A., Sadowski, P.: Generalized open quantum walks on Apollonian networks, vol. 10 (2015)

13. Chia, A., Tan, K., Kurzyński, P., Paterek, T., Kaszlikowski, D., et al.: Coherent chemical kinetics as quantum walks I: Reaction operators for radical pairs. Phys. Rev. E 93(3), 032407 (2016)

14. Whitfield, J.D., Rodríguez-rosario, C.A., Aspuru-guzik, A.: Quantum stochastic walks: A generalization of classical random walks and quantum walks, vol. 81 (2010)

15. Gorini, V., Kossakowski, A., Sudarshan, E.C.G.: Completely positive dynamical semigroups of n-level systems. J. Math. Phys. 17(5), 821-825 (1976)

16. Lindblad, G.: On the generators of quantum dynamical semigroups. Commun. Math. Phys. 48(2), 119130 (1976)

17. Domino, K., Glos, A., Ostaszewski, M., Pawela, Ł., Sadowski, P.: Properties of quantum stochastic walks from the hurst exponent, arXiv preprint (2016)

18. Domino, K., Glos, A., Ostaszewski, M.: Superdiffusive quantum stochastic walk definable of arbitrary directed graph, arXiv:1701.04624 (2017)

19. Szegedy, M.: Quantum Speed-Up of Markov Chain Based Algorithms. In: 2004. Proceedings. 45Th Annual IEEE Symposium On Foundations of Computer Science, pp. 32-41. IEEE (2004)

20. Paparo, G.D., Martin-Delgado, M.: Google in a quantum network. Sci. Rep. 2, 444 (2012)

21. Portugal, R., Santos, R.A., Fernandes, T.D., Gonçalves, D.N.: The staggered quantum walk model. Quantum Inf. Process 15(1), 85-101 (2016)

22. Konno, N., Portugal, R., Sato, I., Segawa, E.: Partition-based discrete-time quantum walks. Quantum Inf. Process 17(4), 100 (2018)

23. Portugal, R., de Oliveira, M.C., Moqadam, J.K.: Staggered quantum walks with hamiltonians. Phys. Rev. A 95(1), 012328 (2017)

24. Tulsi, A.: Robust quantum spatial search. Quantum Inf. Process 15(7), 2675-2683 (2016)

25. Bollobás, B.: Modern graph theory, vol. 184. Springer Science \& Business Media (2013)

26. Hillery, M., Bergou, J., Feldman, E.: Quantum walks based on an interferometric analogy. Phys. Rev. A 68(3), 032314 (2003)

27. Bretto, A.: Hypergraph theory, An introduction. Mathematical Engineering. Springer, Cham (2013)

Publisher's Note Springer Nature remains neutral with regard to jurisdictional claims in published maps and institutional affiliations. 